\title{
BUCHBESPRECHUNGEN
}

William V. O'Brien

\section{The Conduct of Just and Limited War}

Praeger Publishers, New York, N. Y., 1981, 495 S., US $\$ 32.25$

Die Möglichkeit der Führung eines gerechten Krieges auch im Zeitalter der Massenvernichtungsmittel nachweisen zu wollen, erfordert Mut. Ausgerechnet die Intervention der USA in Vietnam als Beispiel für einen solchen anzuführen, grenzt fast an Vermessenheit. Beides aber unternimmt mit spürbarem Engagement, beachtlichem Sachverstand und schlüssiger - wenn auch nicht überall voll überzeugender - Gedankenführung der Georgetown-Professor O'Brien (1956-74 Leiter des Instituts für Weltpolitik dieser Universität) in seinem hier zu besprechenden Werk. Allerdings erscheint ihm ein "gerechter" Krieg nur noch als "begrenzter" Krieg führbar, und schon von daher sollte das Buch nicht nur bei Völkerrechtlern und Politologen, sondern gerade auch bei Politikern und Soldaten Beachtung finden.

In einem einleitenden Kapitel resümiert der Verfasser als Erfahrung seit Ende des Zweiten Weltkriegs, daß Kriege sich wohl nie völlig verhindern lassen würden und begründet damit die Notwendigkeit, ihre Führung auch rechtlich einzugrenzen. Hierzu erscheinen ihm die klassische Figur des gerechten Kriegs und die ergänzende Kategorie des begrenzten Kriegs die geeignetsten Mittel zu sein.

Im sieben Kapitel umfassenden Teil I wendet er sich dann dem gerechten Krieg zu, der sowohl dem Grunde nach (Recht zum Kriege = ius ad bellum: Kap. 2) als auch in seiner Führung (eigentliches Kriegsrecht = ius in bello: Kap. 3) gerecht sein müsse, um dieses Attribut zu verdienen. Der daraus entwickelten These, ein ursprünglich gerechter Krieg könne in seinem Verlauf durch unangemessene Ausweitung der Kriegsziele (ius ad bellum) oder durch unzulässige Methoden der Kriegführung (ius in bello) ungerecht werden, was von den Kriegführenden ständig zu überprüfen sei, wird man wohl uneingeschränkt zustimmen können. Hinsichtlich der vom Autor kasuistisch aufgestellten Gerechtigkeitskriterien kann dies vor allem dort fraglich sein, wo er hinsichtlich des Rechts zum Kriege päpstliche Verlautbarungen wie die Enzyklika "Pacem in Terris" oder die klassischen Schriften des Thomas von Aquino als unmittelbar geltendes Völkerrecht behandelt oder als Erkenntnisquellen für das gegenwärtige ius in bello amerikanische Heeres- und Luftwaffendienstvorschriften (Army Field Manual 27-10; US Air Force Manual AFP 110-31) heranzieht. Allerdings wird man anerkennen müssen, daß diese militärischen Texte wahrscheinlich mehr über die tatsächliche Akzeptanz völkerrechtlicher Kriegsführungsregeln aussagen als die noch so feierliche Ratifizierung entsprechender Abkommen. Diese, insbesondere die einschlägigen Genfer Konventionen, werden natürlich ebenfalls ausführlich herangezogen. Als Ergebnis dieser Erörterungen stellt O’Brien 
fest, daß gerecht nur ein individuell oder kollektiv geführter Verteidigungskrieg - einschließlich Nothilfe durch Dritte, dies aber nur dann, wenn eine realistische Erfolgsaussicht besteht - sein kann, der unter Wahrung des Grundsatzes der Verhältnismäßigkeit der Mittel und unter möglichster Schonung der Zivilbevölkerung geführt wird, wobei nach Möglichkeit Kontakte mit dem Gegner zwecks möglichst frühzeitiger Kriegsbeendigung offenzuhalten sind. Unter keinen Umständen zulässig ist hiernach die "humanitäre Intervention", um eine unterdrückte Bevölkerung von ihrem eigenen Regime zu befreien. $\mathrm{Zu}$ den in diesem Zusammenhang gelegentlich angeführten Interventionen Vietnams in Kampuchea (gegen Pol Pot) und Tanzanias in Uganda (gegen Idi Amin) weist der Verfasser mit Recht darauf hin, daß diese Rechtfertigung von den Interventen selbst nicht in Anspruch genommen wurde.

In den Kapiteln 4 und 5 wendet O'Brien die gewonnenen Kriterien auf die letzten von den USA geführten Kriege an: den Zweiten Weltkrieg, den Koreakrieg und den Vietnamkrieg. Das Eingreifen der USA in den Zweiten Weltkrieg sieht er hinsichtlich Deutschlands - unabhängig von der durch Hitler ausgesprochenen Kriegserklärung - als berechtigte Nothilfe für die angegriffenen europäischen Staaten an. Die später erhobene Forderung der bedingungslosen Kapitulation wertet er dagegen als Exzess, der nur dadurch notdürftig zu rechtfertigen sei, daß es keine erfolgreiche Opposition gegen Hitler gegeben habe, mit der allenfalls über einen Friedensschluß habe verhandelt werden können. Hinsichtlich der Kriegführung erhebt er schwere rechtliche Bedenken gegen die alliierten Flächenbombardierungen ziviler Ziele, ohne die vorangegangenen vergleichbaren deutschen Aktionen (Rotterdam, Coventry) als Rechtfertigung gelten zu lassen: Vergeltung sei kein zulässiger Grund für die Nichtbeachtung der gebotenen Schonung der Nichtkombattanten. Insgesamt gesehen sei die alliierte Kriegführung aber noch als rechtmäßig anzusehen. Im Falle Japans hätten die Japaner mit dem Angriff auf Pearl Harbour den gerechten Kriegsgrund selbst geliefert. Von der Forderung der bedingungslosen Kapitulation sei man gerade noch rechtzeitig abgegangen. Hinsichtlich der Kriegführung gelte das Gleiche wie im Falle Deutschlands. Die beiden Atombombenangriffe auf Hiroshima und Nagasaki müßten allerdings gesondert bewertet werden: Nach heutigem Erkenntnisstand könne man sie nicht rechtfertigen. Man müsse aber akzeptieren, daß nach den damaligen Erkenntnismöglichkeiten die Folgen nicht voll erkennbar gewesen seien und die Einsätze deshalb als Mittel zur schnellen Kriegsbeendigung legitim gewesen seien.

Den koreanischen Fall sieht der Autor mir Recht als relativ einfach an: Der Einsatz zur Rettung Süd-Koreas vor nordkoreanischem Angriff war - unabhängig von der entsprechenden VN-Resolution - als Nothilfe berechtigt. Die vorübergehend erwogene und versuchte Wiedervereinigung Koreas wäre schwieriger zu rechtfertigen gewesen, sei aber nicht zustandegekommen. Die von General MacArthur vorgeschlagene Invasion Chinas (Uberschreitung des Yalu) schließlich sei trotz der Intervention der chinesischen "Volksfreiwilligen" nicht zu rechtf ertigen gewesen und deshalb von der zivilen Führung sofort abgelehnt worden. Bei der Kriegführung sei es zu keinen bedeutenderen Rechtsverletzungen gekommen. Ergebnis: Ein rundum gerechter Krieg. 
Um so vielschichtiger ist die Wertung des Vietnam-Krieges: Die Verteidigung Süd-Vietnams gegen nordvietnamesische Agression sei berechtigt gewesen. Den Bürgerkriegsaspekt des Konflikts erkennt der Autor zwar an, hält ihn aber gegenüber dem Angriff von außen für sekundär. Allenfalls militärisch, kaum aber politisch zu rechtfertigen sei die spätere Einbeziehung von Kambodscha und Laos in die Kampfhandlungen. Auch bei der Kriegführung sei es - teilweise aus kaum zu beseitigenden Zielkonflikten - zu erheblichen Ubergriffen (Entlaubungsaktion, My Lai) gekommen. Insgesamt aber sei der Krieg angesichts der ständigen Verhandlungsbereitschaft der amerikanischen Seite und der bewußten Schonung nordvietnamesischen Territoriums (Bombardierung von Hanoi und Haiphong nur, um Verhandlungsbereitschaft zu erzwingen) doch noch als gerecht einzustufen.

Die drei verbleibenden Kapitel dieses Teils sind der Untersuchung kritischer Probleme eines gerechten Krieges gewidmet: der Nuklearen Abschreckung (Kap. 6) sowie der Anwendung der gewonnenen Kriterien auf einen revolutionären Krieg bzw. von der Gegenseite her betrachtet die Abwehr von Aufstandsbewegungen (counterinsurgency war) (Kap. 7 und 8). Zum ersten Problemkreis stellt der Verfasser einen kaum auflösbaren Konflikt zwischen zwei Erkenntnissen fest: Einerseits müsse die - immerhin seit Jahrzehnten funktionierende-nukleare Abschreckung glaubhaft sein, um wirksam zu bleiben. Andererseits aber könne ein atomarer Erstschlag im Hinblick auf die mutmaßlichen Folgen unter keinen Umständen völkerrechtlich gerechtfertigt werden. Beim revolutionären Krieg sieht er in der sich aus der Natur derartiger Konflikte ergebenden sich gegenseitig ausschließenden Totalität der beiderseitigen Kriegsziele (jeweils Beherrschung des gesamten Staatsgebiets und der gesamten Bevölkerung) die entscheidende Schwierigkeit für die Anwendung völkerrechtlicher Regeln überhaupt. Um so notwendiger sei der Versuch, sie dennoch zu formulieren und durchzusetzen, wie dies im Genfer Protokoll Nr. II von 1977 angestrebt werde.

In den vier Kapiteln des wesentlich kürzeren Teils II wird der begrenzte Krieg abgehandelt. Diesen definiert O'Brien als einen Krieg, der unter politischem Primat mit begrenzten Zielen unter hierauf bezogen proportional begrenztem Kräfteeinsatz und Einhaltung selbstauferlegter Regeln (die kasuistisch aufgeführt werden) geführt wird (Kap. 9). Diese Kriterien wurden, wie er anschließend darlegt, aus dem tatsächlichen Ablauf des Koreakriegs gewonnen, durch den erstmals die Möglichkeit der Führung eines begrenzten Krieges unter modernen Bedingungen als zwar niemanden voll bef riedigende aber doch akzeptable Alternative gegenüber dem totalen Krieg einerseits und der widerstandslosen Hinnahme eines Angriffs andererseits demonstriert worden sei (Kap. 10). In Vietnam führten die USA nach seiner Darstellung zwar einen einwandfrei begrenzten Krieg, aber mit der Situation nicht angemessenen Mitteln (konventionelle Kriegführung in einer Bürgerkriegssituation), verloren dadurch die Unterstützung der Zivilbevölkerung und deshalb schließlich den Krieg (Kap. 11). Als "exemplarisch begrenzter Krieg", zumindest was das Verhalten der beiden Hauptkontrahenten Israel und Ägypten angeht, wird schließlich der Yom-Kippur-Krieg dargestellt (Kap. 12), wobei die totalen Kriegsziele Syriens (Vernichtung Israels) das Kampfgeschehen nicht wesentlich beeinflußt hätten. 
In den beiden Kapiteln (13 und 14) des abschließenden Teils III versucht der Autor, aus den gewonnenen Erkenntnissen realisierbare Richtlinien für das künftige Verhalten der Staaten abzuleiten. Hier bleiben allerdings viele Fragen of fen, und hier vermag die angewandte kasuistische Methode am wenigsten zu befriedigen.

Insgesamt aber liegt hier ein anregendes Werk vor, das die Diskussion einer für den Fortbestand der menschlichen Zivilisation entscheidenden Frage befruchtet und belebt.

Karl Leuteritz

\section{Thomas Darsow}

Zum Wandel des Staatsbegriffs. Unter besonderer Berücksichtigung der Lehre und Praxis internationaler Organisationen, der Mikrostaaten und der PLO

Europäische Hochschulschriften Reihe II, Rechtswissenschaft, Bd. 392, 1984, 288 S., $62,-$ sfr.

Zu den auffälligsten Erscheinungen der modernen Staatenwelt gehört die große Zunahme der sog. "Mikrostaaten" - f rüher mit der deutschen Bezeichnung "Kleinststaaten" belegt - seit der Schlußphase der Dekolonisierung nach 1960. Die völkerrechtliche Literatur, jedenfalls in Deutschland, hat dieses Phänomen bisher eher nur am Rande wahrgenommen. Andere, dringlichere Erscheinungen wie z. B. die internationale Friedenssicherung, die "Neue Weltwirtschaftsordnung", das neue Seerecht oder der Umweltschutz traten in den letzten Jahren in den Vordergrund. Es ist jedoch gleichermaßen ein Trend zu beobachten, sich wieder mehr mit der allgemeinen Völkerrechtstheorie, etwa dem Status Internationaler Organisationen oder der grundsätzlichen Stellung der Staaten im Völkerrecht, zu befassen. So ist es nur zwangsläufig, daß auch der Staatsbegriff des Völkerrechts erneut einer Begutachtung unterzogen wird. Der Arbeit von Darsow kommt so das Verdienst zu, anhand einer Untersuchung über die Mikrostaaten und staatsähnlichen Phänomene (wie der PLO) nicht nur eine völkerrechtsdogmatische Arbeit zu liefern, sondern auch eine Reihe von sonst schwer erreichbaren Informationen über die "großen (kleinen) Unbekannten" unter der Staaten zu vermitteln (etwa in den Fallstudien S. 217 ff.).

Daß die Mikrostaaten trotz ihrer Bedeutungslosigkeit, was Einwohnerzahl oder Staatsfläche anbelangt, in der internationalen Politik eine nicht zu unterschätzende Rolle spielen können, zeigt schon ihr rein zahlenmäßiges Gewicht in den Gremien der Vereinten Nationen, vor allem in der UN-Vollversammlung (S. $168 \mathrm{ff}$.). Die "Atomisierung" des Staates als Völkerrechtssubjekt gibt zu mancherlei Erwägungen Anlaß: Die Kleinstaaten sind heute in der Lage, kraft des Prinzips der formellen rechtlichen Gleichheit der Staaten - wenigstens formal - in gleicher Weise wie eine Großmacht Einfluß auf die Meinungsbildung etwa in der UN-Vollversammlung auszuüben. So stammt z. B. das die internationale wirtschafts- und umweltrechtliche Diskussion beherrschende Schlagwort 